\title{
P53 and Statistical Analysis of the Seminiferous Tubules Epithelium in Experimental Unilateral Abdominal Cryptorchid Testes in Rats
}

F. Al-Bagdadi*, R. Downer**, R. Stout***, T. Gillis****, D. Hillmann*, P. Crawford*, B. Eilts*****

Departments of *Comparative Biomedical Sciences, **Experimental Statistics, ***Laboratory Animal Medicine, $* * * * *$ Veterinary Clinical Sciences and the ****National Hansen's Disease Programs at LSU-SVM, Louisiana State University, Baton Rouge, LA 70803

The frequency of undescended testes has doubled in humans during the last forty years ${ }^{1}$. Cryptorchidism and testicular cancer are associated with impaired spermatogenesis ${ }^{2,3}$. Risks of testicular neoplasms in men with a unilateral abdominal testis were higher than inguinal cryptorchid testis for both the cryptorchid and the scrotal testes ${ }^{3}$. Crytorchidism, the failure of the testes to descend into the scrotum at birth, affects $1 \%$ of newborn boys in the United States ${ }^{4}$, and reports suggest that the incidence is increasing worldwide ${ }^{5}$. The P53-dependent apoptosis appears responsible for cell loss in experimental cryptorchidism ${ }^{6}$. We believe that progressive damage resulting from the cryptorchid testis needs to be statistically documented using P53 as a marker in terms of time. Results could guide the decision for the treatment, prognosis, and the fate of the testes to be either corrected and treated or to be excised.

Twenty-one male sixty-days-old Sprague-Dawley rats from the breeding colony were randomly selected and utilized. All surgical procedures were performed by veterinarians. The rats were divided into four groups, three per group. Unilateral abdominal cryptorchidism of the right testis was induced surgically. Each three rats were sacrificed at 5, 10, 15, and 20 weeks post surgery. Then, each testes was dissected and a $5 \mathrm{~mm}$ thick slice was obtained from the cranial half of each testes. The slices were immersed in Bouin's fixative for 24 hours and dehydrated in graded series of alcohol, cleared in xylene and embedded in paraffin. Five micron thick sections were obtained and stained with Hematoxylin and Eosin and subjected to light microscopic morphometric data collection for statistical analysis. Slices were collected from each testes and placed on dry ice, then homogenized and protein was extracted and estimated, from the homogenates. Western blot was performed using polyclonal anti-P53 antibody. The cell count was obtained from each seminiferous tubule by systematic placement of a 50 point grid randomly over the histological sections at 500X magnification. The mean number of 50 seminiferous tubule cells per testes per rat was averaged and plotted.

The average number of cells per 50 seminiferous tubules per testis decreased progressively in correspondence with the length of the time period of each cryptorchid testis (Figs 1 \& 2), and reached minimum cell average per testis at twenty weeks of cryptorchidism (Fig 3). This indicates that the increase of cellular degeneration by apoptosis and necrosis is progressive as indicated by the decrease in density of the Western blot bands (Fig 4). Further investigation is expected to characterize the time as to wether cryptorchid testes should be corrected or excised.

References

[1] M.B. Jackson, Horm. Res, (1988) 30:153-156.

[2] A. Giwercman et al., J. Urol, (1989) 142:998-1002.

[3] B. Kallen et al., Teratology, (1991) 44: 629-634. 
[4] A. Villumsen et al., Arch Dis Child, (1966) 41:198.

[5] T.K. Jensen et al., Clin HEM, (1995) 41:1896-1901.

[6] Y. Yin et al., J, Androl, (2002) 23: 64-70.

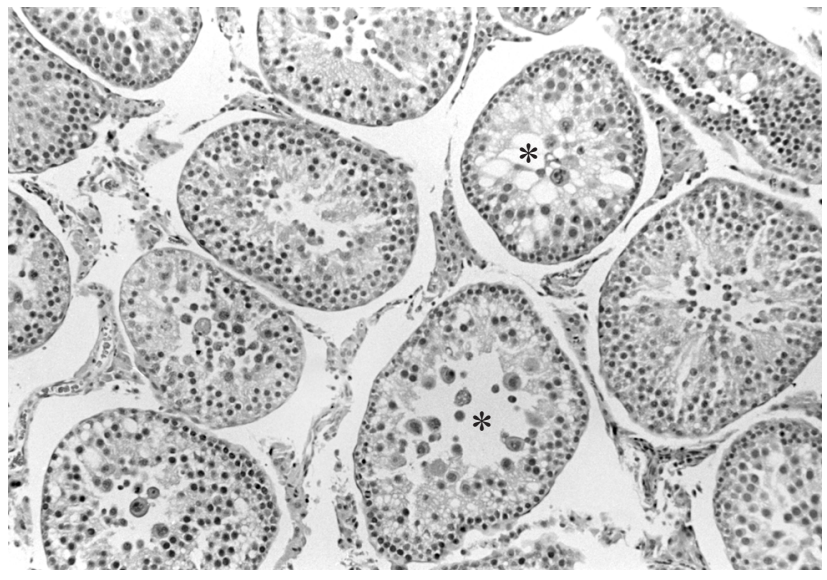

FIG. 1. Several seminiferous tubules* at seven weeks from a unilateral abdominal cryptorchid testis of a rat, show degenerated epithelium by apoptosis. x125.

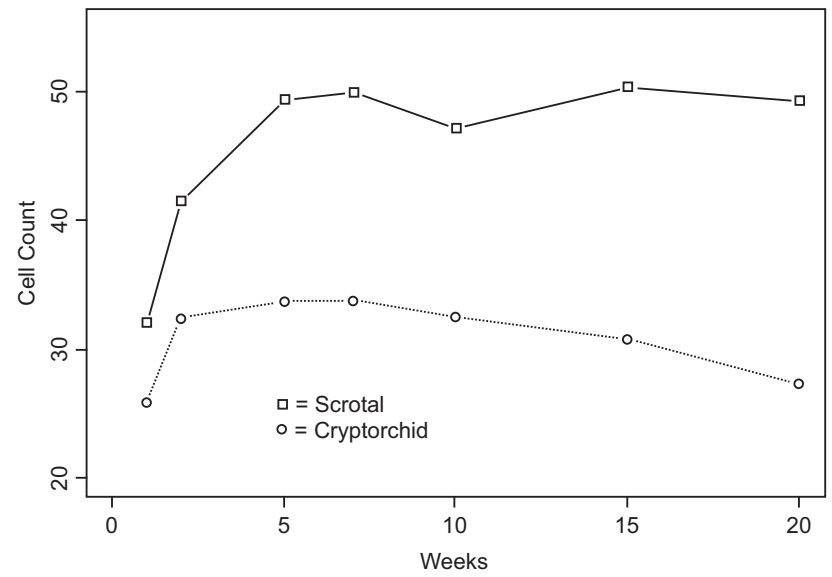

FIG. 3. Scrotal versus cryptorchid testes show weekly progressive cellular apoptosis reaching a minimum at 20 weeks of cryptorchidism.

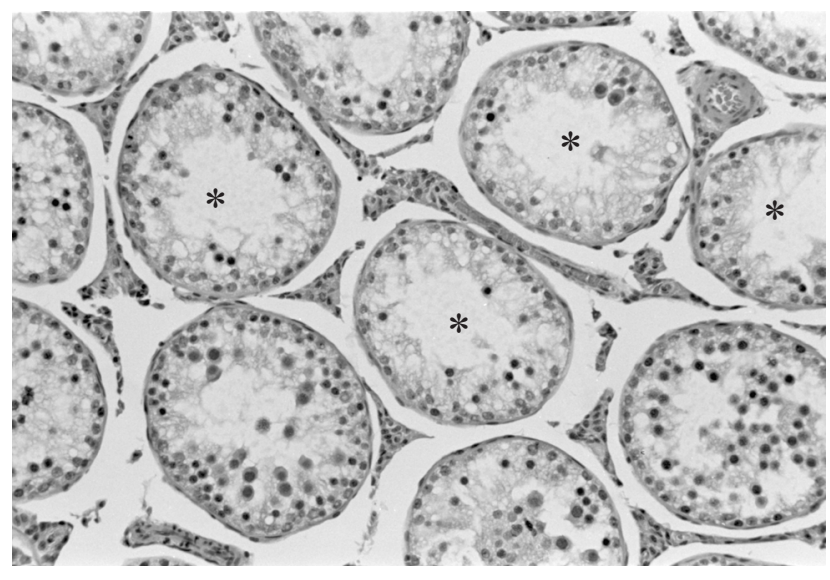

FIG. 2. Most of the seminiferous tubules* at 20 weeks from a unilateral abdominal cryptorchid testis show degenerated epithelium by apoptosis. $\mathrm{x} 128$.

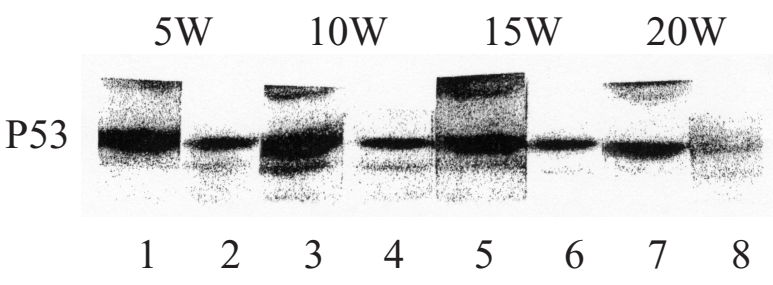

FIG. 4. Western blot of P53 bands from rats with cryptorchid testis for $5 \mathrm{~W}, 10 \mathrm{~W}, 15 \mathrm{~W}$, and 20 weeks. Bands 1, 3, 5, and 7 are from scrotal testes and bands 2, 4, 6, and 8 are from the cryptorchid testes. Band density progressively decreased with time for the cryptorchid testes indicating increased apoptosis. 